\title{
Article
}

\section{Synthesis of graphene nanoribbons by ambient-pressure chemical vapor deposition and device integration}

Zongping Chen, Wen Zhang, Carlos-Andres Palma, Alberto Lodi Rizzini, Bilu Liu, Ahmad N. Abbas, Nils Richter, Leonardo Martini, Xiao-Ye Wang, Nicola Cavani, Hao Lu, Neeraj Mishra, Camilla Coletti, Reinhard Berger, Florian Klappenberger, Mathias Kläui, Andrea

Candini, Marco Affronte, Chongwu Zhou, Valentina De Renzi, Umberto del Pennino,

Johannes V. Barth, Hans Joachim Räder, Akimitsu Narita, Xinliang Feng, and Klaus Müllen

J. Am. Chem. Soc., Just Accepted Manuscript • DOI: 10.1021/jacs.6b10374 • Publication Date (Web): 04 Nov 2016

Downloaded from http://pubs.acs.org on November 7, 2016

\section{Just Accepted}

"Just Accepted" manuscripts have been peer-reviewed and accepted for publication. They are posted online prior to technical editing, formatting for publication and author proofing. The American Chemical Society provides "Just Accepted" as a free service to the research community to expedite the dissemination of scientific material as soon as possible after acceptance. "Just Accepted" manuscripts appear in full in PDF format accompanied by an HTML abstract. "Just Accepted" manuscripts have been fully peer reviewed, but should not be considered the official version of record. They are accessible to all readers and citable by the Digital Object Identifier (DOI®). "Just Accepted" is an optional service offered to authors. Therefore, the "Just Accepted" Web site may not include all articles that will be published in the journal. After a manuscript is technically edited and formatted, it will be removed from the "Just Accepted" Web site and published as an ASAP article. Note that technical editing may introduce minor changes to the manuscript text and/or graphics which could affect content, and all legal disclaimers and ethical guidelines that apply to the journal pertain. ACS cannot be held responsible for errors or consequences arising from the use of information contained in these "Just Accepted" manuscripts. 


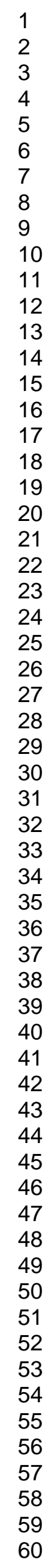

SCHOLARONE"

Manuscripts

7

23

24

26

27

29

30

33

34

35

36

39

40

41

43

44

45

46

47

48

50

51

52

53

54

55

57

58

59

60 


\section{Synthesis of graphene nanoribbons by ambient- pressure chemical vapor deposition and device integration}

Zongping Chen ${ }^{1}$, Wen Zhang ${ }^{1}$, Carlos-Andres Palma ${ }^{3}$, Alberto Lodi Rizzini ${ }^{4,5}$, Bilu Liu $^{6}$, Ahmad Abbas ${ }^{6,7}$, Nils Richter ${ }^{8,9}$, Leonardo Martini ${ }^{4,5}$, Xiao-Ye Wang ${ }^{1}$, Nicola Cavani $^{4,5}$, Hao Lu ${ }^{1}$, Neeraj Mishra ${ }^{10}$, Camilla Coletti ${ }^{10}$, Reinhard Berger ${ }^{2}$, Florian Klappenberger $^{3}$, Mathias Kläui ${ }^{8,9}$, Andrea Candini ${ }^{5}$, Marco Affronte ${ }^{4,5}$, Chongwu Zhou $^{6}$, Valentina De Renzi ${ }^{4,5}$, Umberto del Pennino ${ }^{4,5}$, Johannes V. Barth ${ }^{3}$, Hans Joachim Räder ${ }^{1}$, Akimitsu Narita ${ }^{1}$, Xinliang Feng ${ }^{2 *}$ and Klaus Müllen ${ }^{1 *}$

${ }^{1}$ Max Planck Institute for Polymer Research, Ackermannweg 10, D-55128 Mainz, Germany

${ }^{2}$ Center for Advancing Electronics Dresden (cfaed) \& Department of Chemistry and Food Chemistry, Technische Universität Dresden, Mommsenstrasse 4, 01062 Dresden, Germany

${ }^{3}$ Physik-Department, Technische Universität München, James-Franck-Str. 1, D-85748 Garching, Germany

${ }^{4}$ Dipartimento di Scienze Fisiche, Informatiche e Matematiche, Università di Modena e Reggio Emilia, I-41125 Modena, Italy

${ }^{5}$ CNR-NANO, Istituto Nanoscienze, Centro S3, I-41125 Modena, Italy

${ }^{6}$ Department of Electrical Engineering and Department of Chemistry, University of Southern California, Los Angeles, California, 90089, United States

${ }^{7}$ Department of Electrical Engineering, King Abdulaziz University, Abdullah Sulayman Street, Jeddah 22254, Saudi Arabia

${ }^{8}$ Johannes Gutenberg Universität-Mainz, Institut für Physik, Staudingerweg 7, 55128

Mainz, Germany

${ }^{9}$ Graduate School of Excellence Materials Science in Mainz, Staudingerweg 9, 55128 Mainz, Germany

${ }^{10}$ Center for Nanotechnology Innovation @ NEST, Istituto Italiano di Tecnologia, Piazza San Silvestro 12, 56127 Pisa, Italy

*e-mail: xinliang.feng@tu-dresden.de; muellen@mpip-mainz.mpg.de 
ABSTRACT: Graphene nanoribbons (GNRs), quasi-one-dimensional graphene strips, have shown great potential for nano-scale electronics, optoelectronics and photonics. Atomically precise GNRs can be "bottom-up" synthesized by surfaceassisted assembly of molecular building blocks under ultrahigh vacuum conditions. However, a large-scale and efficient synthesis of such GNRs at low cost remains a significant challenge. Here we report an efficient "bottom-up" chemical vapor deposition (CVD) process for inexpensive and high-throughput growth of structurally defined GNRs with varying structures under ambientpressure conditions. The high quality of our CVD-grown GNRs is validated by a combination of different spectroscopic and microscopic characterizations. Facile, large-area transfer of GNRs onto insulating substrates and subsequent device fabrication demonstrate their promising potential as semiconducting material, exhibiting high current on/off ratio up to 6,000 in field-effect transistor devices. This value is three orders of magnitude higher than values reported so far for other thin film transistors of structurally defined GNRs. Notably, on-surface mass-spectrometry analyses of polymer precursors provide unprecedented evidences for the chemical structures of the resulting GNRs, especially the heteroatom-doping and heterojunctions. These results pave the way toward the scalable and controllable growth of GNRs for future applications.

\section{INTRODUCTION}

Graphene is a two-dimensional semi-metallic crystal with zero bandgap, ${ }^{1-3}$ which hinders its use in many electronic and optoelectronic devices, where a suitable bandgap is essential. ${ }^{4-5}$ It has been predicted by theoretical studies that narrow $(<10$ $\mathrm{nm}$ ) graphene nanoribbons (GNRs) with armchair-type edges exhibit semiconducting behavior, due to the intense quantum confinement and edge effects. ${ }^{6-8}$ Whereas the predominant "top-down" approaches, such as lithographical patterning of graphene ${ }^{9-10}$ and unzipping of carbon nanotubes ${ }^{11-12}$ can hardly control the width and edge structure of GNRs, surface-assisted ${ }^{13-15}$ and solution-mediated ${ }^{16-20}$ "bottom-up" methods have been developed to synthesize atomically precise GNRs. Such "bottomup" synthesized ultranarrow ( 1-2 nm) GNRs have demonstrated large bandgaps of $\sim 1$ to $2 \mathrm{eV}$ with visible to near-infrared absorption, ${ }^{16-23}$ rendering them highly 
interesting for a broad range of applications in next-generation transistors, as well as optoelectronic and photonic devices. ${ }^{24-26}$ However, the device applications of the "bottom-up" synthesized GNRs are still elusive, because on one hand, processing of solution-synthesized GNRs is a major obstacle due to their limited solubility and tendency to aggregate in dispersions. ${ }^{17,19-20,27}$ On the other hand, the amount of GNRs accessible by surface-assisted method is limited, and the elaborate and costly ultrahigh vacuum (UHV) equipment restricts the large-scale fabrication and further use of the GNRs. Sakaguchi and Nakae et al. have previously proposed a use of CVD method for the on-surface synthesis of GNRs, but low pressure was required for the process and unambiguous elucidation of the quality and precise structures of the resulting GNRs has remained elusive. ${ }^{28}$

The precise structures of the GNRs "bottom-up" synthesized under UHV have been demonstrated by high resolution scanning probe microscopy (SPM) together with various spectroscopic characterizations, including Raman spectroscopy and X-ray photoelectron spectroscopy (XPS). ${ }^{13-14,29-32}$ Nevertheless, there is still often a lack of direct evidence for the precise chemical structures of the GNRs. For example, C and $\mathrm{N}$ atoms cannot be directly distinguished in GNRs because of their nearly identical topographic morphology. ${ }^{14,31}$ To date, there is thus no direct evidence for the precise chemical composition of heteroatom-doped GNRs and their heterojunctions. ${ }^{14,30-31,33}$

Here, we demonstrate an efficient ambient-pressure CVD process for inexpensive and high-throughput growth of structurally defined GNRs over large areas, employing hydrogen gas as oxygen scavenger. The GNRs by this CVD method exhibit structural quality and properties comparable with those of the same GNRs synthesized under UHV conditions, as supported by Raman spectroscopy, XPS, high-resolution electron energy loss spectroscopy (HREELS), and scanning tunneling microscopy (STM) characterizations. Homogenous GNR films over areas of square centimeters can be obtained and successfully transferred to non-conducting wafers, allowing for the facile device integration. Thus fabricated field-effect transistor (FET) devices exhibit a large current on/off ratio of up to 6,000, which is significantly higher than values reported so far for thin film transistors made with structurally defined GNRs. ${ }^{27-28,} 34$ Moreover, by using graphene electrodes, the contact resistance can be drastically reduced while preserving the high current on/off ratio. This "bottom-up" CVD method further allows the growth of N-doped and N, S-co-doped GNRs as well as 


\section{RESULTS AND DISCUSSION}

\section{CVD growth of GNRs}

For the growth of chevron-type GNRs, the halogen-functionalized monomer 6,11dibromo-1,2,3,4-tetraphenyltriphenylene (DBTT) was used as molecular precursor (Figs. 1 and S1). In a CVD setup, the DBTT molecules were sublimed by a heating belt and deposited on an Au/mica surface that was kept in the central zone of a horizontal tube furnace at a temperature of $250{ }^{\circ} \mathrm{C}$ (Figs. 1a and S2). The halogen groups of the monomers were removed by thermal activation on the Au surface to form biradical species, which subsequently underwent radical coupling reactions to form covalent $\mathrm{C}-\mathrm{C}$ bonds between each monomer, yielding a chevron-type polyphenylene precursor. Finally, the polyphenylene precursor was annealed to a higher temperature of $450{ }^{\circ} \mathrm{C}$ to establish an extended fully aromatic system, where the surface-assisted intramolecular cyclodehydrogenation afforded the structurally defined chevron-type GNRs (Fig. 1a). In contrast to the UHV ${ }^{13}$ and the three-zone low-pressure $\mathrm{CVD}^{28}$ processes reported before, a two-zone system and ambient pressure were used in our synthesis, for the first time demonstrating that the GNRs can be fabricated efficiently even under such readily available conditions. The results also proved that the surface-assisted polymerization and cyclodehydrogenation reactions for the GNR synthesis are pressure-independent, from UHV to ambient pressure. The CVD growth was carried out under a mixture of $\mathrm{Ar}$ and $\mathrm{H}_{2}$, which is essential to suppress the oxidation of the resulting GNRs as revealed by HREELS and XPS (vide infra). This CVD method can be further applied to different monomers, enabling ambient pressure synthesis of heteroatom-doped GNRs as well as GNR heterojunctions (vide infra). 


\section{Mass spectrometry characterization of the intermediate polyphenylene precursors and GNRs}

The polymerization reaction was first studied by high-resolution matrix-assisted laser desorption/ionization time-of-flight mass spectrometry (MALDI-TOF MS), which is a powerful method to assess the atomic composition of complex organic compounds. ${ }^{35}$ For MALDI-TOF MS measurements, the \{(2E)-2-methyl-3-[4-(2-methyl-2-propanyl) phenyl]-2-propen-1-ylidene \}malononitrile (DCTB) matrix was sublimed on the asdeposited polyphenylene precursors on the Au/mica substrate. The MS spectrum exhibits regular patterns of polyphenylene precursors from dimer $(\mathrm{m} / z=\sim 1,062 \mathrm{Da})$ to pentadecamer $(\mathrm{m} / \mathrm{z}=\sim 7,966 \mathrm{Da})$, in agreement with the expected mass values, which confirms the successful polymerization reaction (Fig. 1e). The observed isotopic distribution is in perfect agreement with the simulated pattern, proving the precise chemical composition of the polyphenylene precursors (Fig. 1e, inset). The intensities of these MS peaks decrease for longer polyphenylene precursors, and precursors larger than fifteen repeating units, i.e., $\sim 12 \mathrm{~nm}$ in length were not observed. We have also successfully investigated the polymerization reaction in CVD growth of other GNRs, such as $N=7$ armchair GNRs (7-AGNRs), using the high-resolution MS analysis method (Fig. S3).

Understanding the nature of the GNR termini is important to reveal the factors limiting the length of GNRs. ${ }^{29}$ There are mainly three plausible GNR termini considering the steps of the CVD growth: radical, $\mathrm{Br}$ and $\mathrm{H}$ passivation. $\mathrm{By}$ combining high-resolution SPM experiments with large-scale density functional theory simulations, Talirz et al. have demonstrated that the termini of GNRs grown under UHV conditions are passivated by hydrogen. ${ }^{29}$ It was suggested that the $\mathrm{H}$ atoms come from premature cyclodehydrogenation in the polymerization step, which can directly compete with the polymerization reaction by forming a $\mathrm{C}-\mathrm{H}$ bond at the radical termini. However, even with those atomically resolved STM analyses, the presence of the hydrogen atoms cannot be directly confirmed. Besides, no SPM investigation has been performed directly on the polyphenylene precursor to reveal its terminal structure in the polymerization reaction. Here, for the first time, we obtained stoichiometric information on the polyphenylene termini by the MALDI-TOF MS analysis as shown in Figs. 1e and S4. No Br terminated polyphenylene precursors can be detected in the samples polymerized at a temperature higher than $250{ }^{\circ} \mathrm{C}$. Besides, 
the exact isotopic distribution of each polyphenylene does not match the radical termini but is in agreement with a H-passivated structure (Fig. S4). These results indeed agree well with the SPM study on GNR termini. ${ }^{29}$ However, no signal that can be attributed to premature cyclodehydrogenation of the precursor can be detected. The amount of premature cyclodehydrogenation might be too limited to be detected by MALDI-TOF MS even if it exists. The reported SPM analysis revealed that $15 \%$ of the GNR termini are methylene groups $\left(\mathrm{H}_{2}\right.$ passivation). ${ }^{29}$ Nevertheless, MS analysis does not support such a termination for the polyphenylene precursors.

Whereas the polyphenylene precursors could be well characterized, the MALDI-TOF MS analysis of the resulting chevron-type GNRs on Au/mica substrates shows no meaningful signals. We suppose that the planar GNRs strongly interacting with the gold surface could not be effectively desorbed by MALDI. ${ }^{17,36}$ When the GNRs were transferred onto a silicon wafer, some carbon fragments could be detected (Fig. S5). Interestingly, all the observed fragments are even-numbered carbon clusters formed through destructive ablation of the GNRs by the laser irradiation in the MS system, which is remarkably similar to the LDI MS spectrum observed for graphene. ${ }^{37}$

\section{UHV characterizations of GNRs}

Although the investigation of ambient-pressure samples under UHV conditions is generally challenging due to the atmospheric contaminants, UHV STM, XPS, and HREELS are key characterization methods for GNRs. Topographical STM surveys of the sample after the first polymerization step show chevron-type polyphenylene precursors (Fig. 1b). High-resolution data reveals zigzag shaped polymer chains in good agreement with that of previous UHV-STM studies, ${ }^{13}$ where the tetraphenyl substitutions marked in green are tilted with respect to the surface, resulting in bright features in the STM images (Fig. 1c, d). After the final cyclodehydrogenation step, a planar chevron-type structure of the resulting GNRs is identified in both ambient and UHV STM investigations (Fig. 2a-c). Higher resolution UHV image reveals additional imperfections at the submolecular level. The white and cyan arrows in Fig. $2 \mathrm{~b}$ indicate common defects due to broken phenyl rings and randomly coupled GNRs, respectively. To be noted is that even the "atomically precise" GNR made under UHV are not always $100 \%$ atomically precise, in terms of the curving and branching. ${ }^{13}$ Recently, the coupling of several GNRs into complex junction structures has also 
been found in the UHV-grown GNRs and revealed by CO-functionalized atomic force microscopy in atomic resolution. ${ }^{38-39}$ These coupling junctions between GNRs may be important tool in the development of graphene-based circuitry and facilitate the electronic device investigation on GNRs. ${ }^{38}$ Large scale survey was difficult to obtain due to adsorbed impurities from ambient pressure condition (bright white protrusions in Fig. 1b-d and Fig. 2a-c), hindering quantitative statistical analysis of the GNR quality. Nevertheless, we would like to highlight that we can synthesize GNRs much faster and facilely under the CVD conditions with a significantly simpler setup, which can be easily scaled up for applications. We suggest that further preparation of the samples in clean room conditions and vacuum packaging may improve the quality of GNRs and also facilitate the UHV STM characterization.

Instead, the quality of the CVD-grown chevron-type GNRs was further examined by comparing the XPS and HREELS spectra with those of a reference GNR sample, which was grown in-situ under UHV conditions on an Au(111) single crystal ${ }^{13}$ (Figs. $2 \mathrm{e}$ and $\mathrm{f}$ ). The overlayer coverage, as estimated from the intensity of the $\mathrm{C} 1 \mathrm{~s}$ peak relative to the Au 4f peak in the XPS spectrum, is very similar for both samples, corresponding to 0.5 ML for the UHV-grown GNRs and 0.6 ML for the CVD-grown GNRs (1 ML is defined as a single GNR layer with surface number density of $2.7 \times 10^{15}$ at. $/ \mathrm{cm}^{2}$, see the Supplementary Information for details). The XPS survey spectra exhibit only the core level peaks belonging to the GNRs and gold substrate. The C1s core level spectrum of the CVD-grown GNRs consists of a single sharp component located at $\sim 284 \mathrm{eV}$ binding energy, corresponding to the $s p^{2}$ bonded carbon, which well resembles that of the UHV-grown GNRs (Fig. 2e). No Br components can be detected, indicating that $\mathrm{Br}$ atoms are completely desorbed from the gold surface. HREELS can precisely characterize the vibrational properties of the overlayer, serving as a powerful characterization method for GNR thin films especially when IR absorption is too weak for a reasonable analysis. ${ }^{30,} 40$ The HREELS spectra of the two samples are almost identical: both are indeed dominated by the out-of-plane $\mathrm{CH}$ mode manifold, located in the $700-850 \mathrm{~cm}^{-1}$ region, while the intensity of the in-plane $\mathrm{CH}$ stretching mode at $3,063 \mathrm{~cm}^{-1}$ is almost negligible (Fig. 2f). The manifold is built up by four main peaks locates at 754, 782, 806, and $862 \mathrm{~cm}^{-}$ 1 , which are assignable to out-of-plane modes $\gamma(\mathrm{C}-\mathrm{H})$, characteristic of different molecular edge topologies (i.e., TRIO, DUO and SOLO structures in Refs. ${ }^{41-42}$ ). It is 
important to notice that in both spectra the feature at $\sim 700 \mathrm{~cm}^{-1}$, which is attributed to the out-of-plane $\gamma(\mathrm{C}-\mathrm{H})$ mode of the mono-substituted benzene rings and is therefore present only for the monomer and polyphenylene precursors, is indeed barely visible. ${ }^{30}$ This finding, together with the dominant intensity of out-of-plane modes relative to in-plane ones, proves that the reaction from the polyphenylene precursors to flat GNRs is completed. ${ }^{30}$ The quality of the CVD-grown GNRs is also documented by the huge intensity of the elastic peak, and the narrow width of the vibrational modes, which are comparable with those of the UHV-grown GNRs. The slight differences seen in the spectra might be caused by the atmospheric contaminants on the $\mathrm{Au}(111)$ surface under the ambient-pressure conditions. Besides, compared with UHV conditions, we have found that hydrogen is important for CVD growth of high quality GNRs. Normally, the oxygen content in CVD is much larger than the UHV system and is inevitable, leading to the considerable oxidation of the resulting GNRs, as evidenced by the HREELS analysis: The HREELS spectra of the CVD-GNRs grown without $\mathrm{H}_{2}$ displays two extra broad features located at 440 and $1,100 \mathrm{~cm}^{-1}$, which may attribute to C-O-C deformation and stretching modes (Fig. 2f). The oxidation of the GNRs grown without $\mathrm{H}_{2}$ was further corroborated by XPS where much higher level of oxygen content was detected (see Fig. S6 and the Supplementary Information for details). High quality of the CVD-grown GNRs could be achieved only under the presence of $\mathrm{H}_{2}$ in the growth, which can suppress the oxidation of the GNRs during the high temperature annealing (Fig. 2f). Therefore, combination of the high-resolution STM, HREELS, and XPS analyses proved that structurally-defined GNRs can be synthesized by our CVD method under the presence of $\mathrm{H}_{2}$, with high quality comparable to that of the UHV-grown GNRs.

\section{Transfer of the GNRs and their Raman and UV-Vis-NIR spectroscopy characterizations}

The Raman spectra of the CVD-grown chevron-type GNRs excited by a $532 \mathrm{~nm}$ laser is presented in Fig. 2d and Fig. S7, where the most intense peaks are at $\sim 1,330$ and $1,608 \mathrm{~cm}^{-1}$, typically assigned to D and G bands, respectively. ${ }^{43}$ Remarkably, sharp peaks of low-frequency modes can be observed at $\sim 180, \sim 284$, and $\sim 403 \mathrm{~cm}^{-1}$, which may be related to the specific GNR structure, like the width-specific radial breathinglike mode (RBLM), ${ }^{13}{ }^{19}$ indicating high uniformity of the chevron-type GNRs. Raman spectrum of the CVD-grown 7-AGNRs demonstrates perfect agreement with that of 
the same GNR prepared under the UHV condition, including the RBLM at $\sim 400 \mathrm{~cm}^{-1}$ and other smaller features, which further corroborates the highly defined structures of the CVD-grown GNRs (Fig. S3).

Moreover, the quantity of GNRs and the size of the resulting films can be easily scaled up by using large Au/mica substrates, e.g., with an area of $25 \times 75 \mathrm{~mm}^{2}$ as shown in Fig. 3a (essentially limited only by the furnace and tube dimensions), which is much larger than the reported small piece of GNRs by CVD (limited to a size of $\sim 10 \times 10 \mathrm{~mm}^{2}$ ) using the similar size of quartz tube. ${ }^{28}$ After the CVD growth, the GNRs can be transferred onto other substrates with or without the assistance of poly(methyl methacrylate) (PMMA) (Scheme S2 and S3). The transferred GNR films possess a high uniformity over an area of square centimeters without any cracks based on the optical images and Raman mapping (Figs. 3b and S7-9). Atomic force microscopy of the GNRs transferred on silicon wafer reveals a thickness of $\sim 0.7 \mathrm{~nm}$ (Fig. 3d). The chevron-type GNR films have also been transferred layer by layer on fused silica for UV-Vis-NIR absorption measurements (Fig. S10). The spectrum exhibits absorption in the UV and visible region with an absorption maximum at $\sim 450$ $\mathrm{nm}$. The optical bandgap of the GNR film was roughly estimated from the absorption onset at $\sim 700 \mathrm{~nm}$ to be approximately $1.8 \mathrm{eV},{ }^{19-20}$ suggesting their potential for electronic device applications.

\section{Electronic properties of GNR-based three-terminal FET devices}

Based on the successful transfer of chevron-type GNR films on any desired substrates, we have fabricated three-terminal FET devices using GNRs as the channel material, to study their charge transport properties. The first set of devices was fabricated from chevron-type GNR films transferred on a silicon wafer, where Pd/Ti $(50 \mathrm{~nm} / 1 \mathrm{~nm})$ electrodes were patterned as the contact metals using electron-beam lithography (EBL) (Fig. 3c). The channel length was $\sim 1-2 \mu \mathrm{m}$ (see Supplementary Information for more details). As shown in Figs. 3e-f and S11a, a large gate-modulation to the source-drain current can be observed (current on/off ratio >1,000). The GNR transistors exhibit a unipolar p-type conduction, as the source-drain current increases with increasing negative gate voltages. The p-type conduction might be caused by doping via adatoms (e.g. oxygen from air), since the device measurements were carried out in ambient condition. $^{44}$ Best performing devices exhibit current on/off ratio of $>6,000$, which is 
significantly higher (more than three orders of magnitude) than the values reported so far for other "bottom-up" synthesized GNR thin film network transistors (with typical on/off ratio of $2-5),{ }^{27-28,34}$ and even better than the devices on UHV-GNRs with an ultranarrow channel. ${ }^{44}$ Fig. $3 \mathrm{f}$ shows $I_{\mathrm{ds}}-V_{\mathrm{ds}}$ curves of a typical GNR transistor. The non-linear nature of the curves at small bias regimes suggests Schottky-contact behavior. This observation is understandable because our GNRs are very narrow and possess a large bandgap. Since the GNR devices reveal p-type conduction in air, the use of high work function contact materials, such as $\mathrm{MoO}_{3-\mathrm{x}},{ }^{45}$ may offer better contacts to the valence band of GNRs and deliver better device performance.

Alternatively, reducing the channel length may also improve the device performance, as the additional resistance between adjacent ribbons is reduced or can be completely avoided. This has been tested in an additional set of devices where the Pd electrode was in direct contact with the GNR films and the spacing between the electrodes was smaller than $50 \mathrm{~nm}$ (Fig. 4a). Because of the drastically reduced active channel length, the same level of current flow can be achieved at one order of magnitude lower source-drain voltages. However, in that case the influence of the gate voltage is drastically reduced, which may be attributed to the screening effect of the metal electrodes on the electric field from the gate. Therefore, either a thinner insulating barrier layer or a better source-drain electrode material would be needed to retain the FET-functionality in the short-channel devices. For this purpose, and by taking advantage of the facile transfer process of our GNRs, we realized another type of three-terminal FET device by direct transfer of the chevron-type GNRs on prefabricated graphene electrodes, i.e. a graphene device where the channel has been opened with a gap of approximately $100 \mathrm{~nm}$ by EBL and oxygen plasma etching (see Fig. $4 \mathrm{~b}$ and the Supplementary Information for details). Relatively thick graphene (average 10 layers) on $\mathrm{C}$-face of $\mathrm{SiC}$ is chosen as the electrode material, which displays almost no gate dependence and is expected to show limited screening of the gate potential compared to metal electrodes. The electrical characteristics of the resulting devices are presented in Fig. 4c-d (see Fig. S11-13 and the Supplementary Information for more details). The contact resistance is greatly reduced with respect to the long-channel metal electrodes, due to the shorter channel distance and a superior GNR/graphene interface. An evident transistor effect (current vs side-gate voltage) can be observed with a current on/off ratio of $>100$, limited only by the maximum 
applicable gate voltage. Improvement of the channel-gate coupling, for instance by employing a top gate with a thin high- $k$ dielectric, is expected to further enhance the device performances. Interestingly, contrary to the p-type conduction observed in the long-channel devices, the devices based on graphene electrodes show an n-type conduction, which was previously found also for the UHV-GNR device. ${ }^{44}$ The $I_{\mathrm{ds}}-V_{\mathrm{ds}}$ curves in Fig. 4d remain non-linear, indicating the presence of a Schottky-type contact also in these devices. We suggest that the performance of these GNR devices can be further improved by using hBN as its support. Moreover, development of wider GNRs with smaller bandgaps and growth of longer GNRs through optimization of the CVD process may further reduce the Schottky barriers and contact resistance for a better electrical connection to electrodes.

\section{Heteroatom-doped GNRs and GNR heterojunctions}

The "bottom-up" CVD procedure can be a general strategy for fabricating a broad class of GNRs and their hybrids with engineered structures and properties based on the selection of suitable monomers. As an example, we have demonstrated the CVD growth of heteroatom-doped chevron-type GNRs, which is a promising way to modify the electronic structures of GNRs. ${ }^{14,30}$ By using the nitrogen and sulphur containing monomers 4,4'-(6,11-dibromo-1,4-diphenyltriphenylene-2,3diyl)dipyridine (DBNN) and 4-(6,11-dibromo-1,4-diphenyl-3-(thien-3-yl)triphenylen2-yl)pyridine (DBNS, Fig. 5 and Scheme S1) as precursors, N-doped and N, S-codoped chevron-type GNRs can be grown in the CVD system, respectively (Scheme S4). High-resolution MALDI-TOF MS analysis clearly validates the polymerization of the corresponding dehalogenated biradical intermediates, forming $\mathrm{N}$-substituted and N, S-co-substituted polyphenylene precursors (Figs. 5b, e and S14-15). Contrary to symmetric monomer DBNN, monomer DBNS has an asymmetric structure with both pyridyl and thienyl groups. Therefore, it must be noted that the resulting N, S-cosubstituted polyphenylene precursors always contain regioisomeric segments, depending on the head-to-head or head-to-tail coupling, which can only be differentiated by atomic-resolution SPM. The successful cyclodehydrogenation of these precursors into the N-doped and N, S-co-doped GNRs is corroborated by Raman spectroscopy (Fig. S16). The observation of intense Raman spectra with the sharp $\mathrm{G}$ peak located at $\sim 1,608 \mathrm{~cm}^{-1}$ and $\mathrm{D}$ peak located at $\sim 1,330 \mathrm{~cm}^{-1}$ validated the successful "graphitization" of the polymer precursors into GNR structures. We have 
also performed XPS and HREELS analyses of the N- and N, S-co-doped GNRs grown by our CVD method (see Figs. S17-18 and the Supplementary Information for details).

We further fabricated GNR heterojunctions in the CVD system by co-sublimation of different kinds of structurally complementary monomers. Figure 5a illustrates the strategy for fabricating a p-N-GNR heterojunctions with seamlessly connected segments of pristine (p) and N-doped (N) GNRs. ${ }^{14}$ The monomer DBTT and its Nsubstituted equivalent DBNN were co-deposited on an $\mathrm{Au} / \mathrm{mica}$ substrate kept at $200{ }^{\circ} \mathrm{C}$, resulting in polyphenylene heterojunctions, which could be directly identified by the MS analysis (Figs. 5a, c, S19 and Scheme S4). The deposited radical intermediates of the two monomers distribute randomly and uniformly on the gold surface. ${ }^{15}$ Therefore, the building blocks in the hybrid polyphenylene precursors are mixed and fused in a random sequence during the polymerization, exhibiting a statistical distribution from pure segments of each monomer unit to their combinations with different ratio. For example, the isotopic distributions of the resulted trimeric polyphenylene precursors exhibit four kinds of trimer structures, with the ratio of the two monomer units varying from $3 / 0,2 / 1,1 / 2$ to $0 / 3$, where the middle two structures are corresponding to the polyphenylene heterojunctions (Fig. 5c). Moreover, the CVD method allows for the synthesis of polyphenylene heterojunctions with various chemical compositions simply by using different monomers. For example, heterojunctions of N-substituted and N, S-co-substituted polyphenylenes can be fabricated by combining the $\mathrm{N}$ - and $\mathrm{N}, \mathrm{S}$-co-substituted monomers (DBNN and DBNS), which have been unambiguously identified by highresolution MS analysis (Figs. 5f and S20). These results mark the high versatility of the CVD process and the great effectiveness of this direct MS analysis method for investigating the GNR growth process. All these polyphenylene heterojunctions can be converted to GNR heterojunctions similarly by cyclodehydrogenation upon further thermal annealing at $400-420{ }^{\circ} \mathrm{C}$, as verified by Raman spectroscopic analyses (Fig. S16). 


\section{CONCLUSION}

In summary, the low-cost CVD method has enabled the large-scale "bottom-up" growth of structurally-defined pristine GNRs and selectively N-doped and N, S-codoped GNRs as well as their heterojunctions at the ambient pressure, which can be an excellent complement to the more elaborate UHV method. Raman, HREELS, XPS, and STM analyses manifested the high quality of the CVD-grown GNRs well comparable to those grown under UHV conditions. The surface-mass-spectrometry analysis can serve as a quick and powerful method to investigate the precise chemical structures of various GNR precursors as well as other surface-bound molecular materials. Further studies might also provide an insight into the reaction mechanisms of the surface-based polymerization and cyclodehydrogenation. Large-area GNR films can be readily transferred on to arbitrary substrates, where preliminary micro- as well as nano-scale transistor devices could be fabricated, demonstrating unprecedentedly strong gate modulation for the "bottom-up" GNRs. Furthermore, our large-scale synthesis process can be readily applied to other designed monomers for preparing GNRs with engineered topographic and chemical structures, which is expected to provide practical solutions to the current challenges in graphene-based nanoelectronic, optoelectronic and photonic devices. 


\section{EXPERIMENTAL SECTION}

CVD growth of GNRs. Home-made and commercially available (Phasis, Switzerland and Georg Albert, Germany) $200 \mathrm{~nm}$ Au thin films epitaxially grown on mica were used as substrates for GNR growth. They were cut into pieces with sizes from $10 \times 10$ to $25 \times 75 \mathrm{~mm}^{2}$ and placed in a quartz tube of outer diameter $35 \mathrm{~mm}$ and inner diameter $33 \mathrm{~mm}$ in a horizontal tube furnace (Nabertherm, RT 80-250/11S). Precursor monomers (for details on the synthesis, see the Supplementary Information) were loaded upstream of the Au/mica substrates and sublimed at $250-325{ }^{\circ} \mathrm{C}$ onto the Au/mica surface using a heating belt (Thermocoax Isopad S20). The Au/mica substrates were heated and maintained at $200-250{ }^{\circ} \mathrm{C}$ under gas flow of Ar (500 s.c.c.m.) and $\mathrm{H}_{2}$ (100 s.c.c.m.) during monomer sublimation for 5-30 min to induce dehalogenation and radical addition reaction. After deposition, the samples were postannealed at $400-450{ }^{\circ} \mathrm{C}$ for $15 \mathrm{~min}$ to convert the polyphenylene precursors into GNRs by cyclodehydrogenation. For fabrication of the heterojunctions, the two precursor species were evaporated together onto an $\mathrm{Au} / \mathrm{mica}$ substrate held at $200{ }^{\circ} \mathrm{C}$, which was subsequently annealed to $400-420{ }^{\circ} \mathrm{C}$. To study the oxidation of the GNRs, only 500 s.c.c.m. of Ar was used in the growth without $\mathrm{H}_{2}$, the pressure was $\sim 0.7$ mbar by a mechanical rotary pump (capable of low pressure down to $<10^{-4} \mathrm{mbar}$ ).

Transfer of GNRs. As-grown GNRs/Au/mica substrates were placed on a surface of hydrofluoric acid (HF) (40 wt. \%) solution, which etched away the mica and made the $\mathrm{Au}$ film with GNRs float on the surface of the HF solution. After washing with ultrapure water, the GNRs/Au films were transferred to a new insulated substrate and dried at $100{ }^{\circ} \mathrm{C}$ for 10 minutes. Finally, the $\mathrm{Au}$ films were etched away by gold etchant (Sigma-Aldrich), leaving only the GNR films on the new substrate. When transferring GNR films with large areas, spin-coated PMMA thin films were used as a mechanical support before etching with HF to avoid breaking the GNR films. The PMMA was washed away by hot acetone in the last step (Scheme S2 and S3). 


\section{ASSOCIATED CONTENT}

\section{Supporting Information}

Experimental details, MALDI-TOF MS, Raman spectra, UV-vis spectra, more analysis on XPS and HREELS spectra of the GNRs and their FET devices. The Supporting Information is available free of charge on the ACS Publications website.

\section{AUTHOR INFORMATION \\ Corresponding Authors \\ *xinliang.feng@tu-dresden.de \\ *muellen@mpip-mainz.mpg.de}

\section{Notes}

The authors declare no competing financial interest.

\section{ACKNOWLEDGMENTS}

This work was supported by the European Research Council grant on NANOGRAPH and MolArt, DFG Priority Program SPP 1459, MIUR FIR grant RBFR13YKWX and project PRIN-GRAF 20105ZZTSE_008, Graphene Flagship (No. CNECT-ICT604391), European Union Projects UPGRADE and MoQuaS (contract N.610449), and the Office of Naval Research BRC Program. The authors thank Dr. Roberto Biagi and Valdis Corradini (CNR-NANO, Istituto Nanoscienze) for useful discussions, and Dr. Dieter Schollmeyer (Institute for Organic Chemistry, Johannes Gutenberg University Mainz) for single-crystal X-ray structural analysis of the monomer DBNS. X.Y.W. is grateful for the fellowship from Alexander von Humboldt Foundation. 


\section{REFERENCES}

(1) Novoselov, K. S.; Geim, A. K.; Morozov, S. V.; Jiang, D.; Zhang, Y.; Dubonos, S. V.; Grigorieva, I. V.; Firsov, A. A. Science 2004, 306, 666-669.

(2) Geim, A. K. Science 2009, 324, 1530-1534.

(3) Geim, A. K.; Novoselov, K. S. Nature Mater. 2007, 6, 183-191.

(4) Kotov, V. N.; Uchoa, B.; Pereira, V. M.; Guinea, F.; Castro Neto, A. H. Rev. Mod. Phys. 2012, 84, 1067-1125.

(5) Castro Neto, A. H.; Guinea, F.; Peres, N. M. R.; Novoselov, K. S.; Geim, A. K. Rev. Mod. Phys. 2009, 81, 109-162.

(6) Yang, L.; Park, C. H.; Son, Y. W.; Cohen, M. L.; Louie, S. G. Phys. Rev. Lett. 2007, 99, 186801.

(7) Barone, V.; Hod, O.; Scuseria, G. E. Nano Lett. 2006, 6, 2748-2754.

(8) Palma, C.-A.; Awasthi, M.; Hernandez, Y.; Feng, X.; Müllen, K.; Niehaus, T. A.; Barth, J. V. J. Phys. Chem. Lett. 2015, 6, 3228-3235.

(9) Chen, Z.; Lin, Y.-M.; Rooks, M. J.; Avouris, P. Physica E 2007, 40, 228-232.

(10) Han, M. Y.; Ozyilmaz, B.; Zhang, Y.; Kim, P. Phys. Rev. Lett. 2007, 98, 206805.

(11) Jiao, L. Y.; Zhang, L.; Wang, X. R.; Diankov, G.; Dai, H. J. Nature 2009, 458, 877-880.

(12) Kosynkin, D. V.; Higginbotham, A. L.; Sinitskii, A.; Lomeda, J. R.; Dimiev, A.; Price, B. K.; Tour, J. M. Nature 2009, 458, 872-876.

(13) Cai, J. M.; Ruffieux, P.; Jaafar, R.; Bieri, M.; Braun, T.; Blankenburg, S.; Muoth, M.; Seitsonen, A. P.; Saleh, M.; Feng, X. L.; Müllen, K.; Fasel, R. Nature 2010, 466, 470-473.

(14) Cai, J. M.; Pignedoli, C. A.; Talirz, L.; Ruffieux, P.; Söde, H.; Liang, L. B.; Meunier, V.; Berger, R.; Li, R. J.; Feng, X. L.; Müllen, K.; Fasel, R. Nature Nanotech. 2014, 9, 896-900.

(15) Chen, Y. C.; Cao, T.; Chen, C.; Pedramrazi, Z.; Haberer, D.; de Oteyza, D. G.; Fischer, F. R.; Louie, S. G.; Crommie, M. F. Nature Nanotech. 2015, 10, 156-160.

(16) Dössel, L.; Gherghel, L.; Feng, X.; Müllen, K. Angew. Chem. Int. Ed. 2011, 50, 2540-2543.

(17) Schwab, M. G.; Narita, A.; Hernandez, Y.; Balandina, T.; Mali, K. S.; De Feyter, S.; Feng, X.; Müllen, K. J. Am. Chem. Soc. 2012, 134, 18169-18172.

(18) Yang, X.; Dou, X.; Rouhanipour, A.; Zhi, L.; Rader, H. J.; Müllen, K. J. Am. Chem. Soc. 2008, 130, 4216-4217.

(19) Narita, A.; Feng, X.; Hernandez, Y.; Jensen, S. A.; Bonn, M.; Yang, H.; Verzhbitskiy, I. A.; Casiraghi, C.; Hansen, M. R.; Koch, A. H.; Fytas, G.; Ivasenko, O.; Li, B.; Mali, K. S.; Balandina, T.; Mahesh, S.; De Feyter, S.; Müllen, K. Nature Chem. 2014, 6, 126-132.

(20) Vo, T. H.; Shekhirev, M.; Kunkel, D. A.; Morton, M. D.; Berglund, E.; Kong, L.; Wilson, P. M.; Dowben, P. A.; Enders, A.; Sinitskii, A. Nature Commun. 2014, 5, 3189.

(21) Narita, A.; Verzhbitskiy, I. A.; Frederickx, W.; Mali, K. S.; Jensen, S. A.; Hansen, M. R.; Bonn, M.; De Feyter, S.; Casiraghi, C.; Feng, X.; Müllen, K. ACS Nano 2014, 8, 11622-11630.

(22) Chen, L.; Hernandez, Y.; Feng, X.; Müllen, K. Angew. Chem. Int. Ed. 2012, 51,7640-7654. 
(23) Narita, A.; Wang, X. Y.; Feng, X. L.; Müllen, K. Chem. Soc. Rev. 2015, 44, 6616-6643.

(24) Lee, S. J.; Kim, J.-Y.; Kim, H. P.; Kim, D.; da Silva, W. J.; Schneider, F. K.; Mohd Yusoff, A. R. b.; Jang, J. Chem. Commun. 2015, 51, 9185-9188.

(25) Osella, S.; Narita, A.; Schwab, M. G.; Hernandez, Y.; Feng, X.; Müllen, K.; Beljonne, D. ACS Nano 2012, 6, 5539-5548.

(26) Bonaccorso, F.; Sun, Z.; Hasan, T.; Ferrari, A. C. Nature Photonics 2010, 4, 611-622.

(27) Abbas, A. N.; Liu, G.; Narita, A.; Orosco, M.; Feng, X. L.; Müllen, K.; Zhou, C. W. J. Am. Chem. Soc. 2014, 136, 7555-7558.

(28) Sakaguchi, H.; Kawagoe, Y.; Hirano, Y.; Iruka, T.; Yano, M.; Nakae, T. Adv. Mater. 2014, 26, 4134-4138.

(29) Talirz, L.; Söde, H.; Cai, J.; Ruffieux, P.; Blankenburg, S.; Jafaar, R.; Berger, R.; Feng, X.; Müllen, K.; Passerone, D.; Fasel, R.; Pignedoli, C. A. J. Am. Chem. Soc. 2013, 135, 2060-2063.

(30) Bronner, C.; Stremlau, S.; Gille, M.; Brausse, F.; Haase, A.; Hecht, S.; Tegeder, P. Angew. Chem. Int. Ed. 2013, 52, 4422-4425.

(31) Zhang, Y.; Zhang, Y. F.; Li, G.; Lu, J. C.; Lin, X.; Du, S. X.; Berger, R.; Feng, X. L.; Müllen, K.; Gao, H. J. Appl. Phys. Lett. 2014, 105, 023101.

(32) Batra, A.; Cvetko, D.; Kladnik, G.; Adak, O.; Cardoso, C.; Ferretti, A.; Prezzi, D.; Molinari, E.; Morgante, A.; Venkataraman, L. Chem. Sci. 2014, 5, 4419-4423.

(33) Vo, T. H.; Shekhirev, M.; Kunkel, D. A.; Orange, F.; Guinel, M. J. F.; Enders, A.; Sinitskii, A. Chem. Commun. 2014, 50, 4172-4174.

(34) Zschieschang, U.; Klauk, H.; Mueller, I. B.; Strudwick, A. J.; Hintermann, T.; Schwab, M. G.; Narita, A.; Feng, X. L.; Müllen, K.; Weitz, R. T. Adv. Electron. Mater. 2015, $1,1400010$.

(35) Hillenkamp, F.; Peter-Katalinic, J., MALDI MS: a practical guide to instrumentation, methods and applications. John Wiley \& Sons: 2013.

(36) Palma, C. A.; Diller, K.; Berger, R.; Welle, A.; Björk, J.; Cabellos, J. L.; Mowbray, D. J.; Papageorgiou, A. C.; Ivleva, N. P.; Matich, S.; Margapoti, E.; Niessner, R.; Menges, B.; Reichert, J.; Feng, X.; Rader, H. J.; Klappenberger, F.; Rubio, A.; Müllen, K.; Barth, J. V. J. Am. Chem. Soc. 2014, 136, 4651-4658.

(37) Kong, X.; Huang, Y.; Chen, Y. J. Mass. Spectrom. 2012, 47, 523-528.

(38) Dienel, T.; Kawai, S.; Söde, H.; Feng, X.; Müllen, K.; Ruffieux, P.; Fasel, R.; Gröning, O. Nano Lett. 2015, 15, 5185-5190.

(39) Kawai, S.; Saito, S.; Osumi, S.; Yamaguchi, S.; Foster, A. S.; Spijker, P.; Meyer, E. Nature Commun. 2015, 6, 8098.

(40) Bronner, C.; Leyssner, F.; Stremlau, S.; Utecht, M.; Saalfrank, P.; Klamroth, T.; Tegeder, P. Phys. Rev. B 2012, 86, 085444.

(41) Centrone, A.; Brambilla, L.; Renouard, T.; Gherghel, L.; Mathis, C.; Müllen, K.; Zerbi, G. Carbon 2005, 43, 1593-1609.

(42) Tommasini, M.; Lucotti, A.; Alfè, M.; Ciajolo, A.; Zerbi, G. Spectrochimica Acta A 2016, 152, 134-148.

(43) Ferrari, A. C.; Meyer, J. C.; Scardaci, V.; Casiraghi, C.; Lazzeri, M.; Mauri, F.; Piscanec, S.; Jiang, D.; Novoselov, K. S.; Roth, S.; Geim, A. K. Phys. Rev. Lett. 2006, 97, 187401.

(44) Bennett, P. B.; Pedramrazi, Z.; Madani, A.; Chen, Y. C.; de Oteyza, D. G.; Chen, C.; Fischer, F. R.; Crommie, M. F.; Bokor, J. Appl. Phys. Lett. 2013, 103, 253114. 
(45) Chuang, S.; Battaglia, C.; Azcatl, A.; McDonnell, S.; Kang, J. S.; Yin, X. T.; Tosun, M.; Kapadia, R.; Fang, H.; Wallace, R. M.; Javey, A. Nano Lett. 2014, 14, 1337-1342. 
Figures

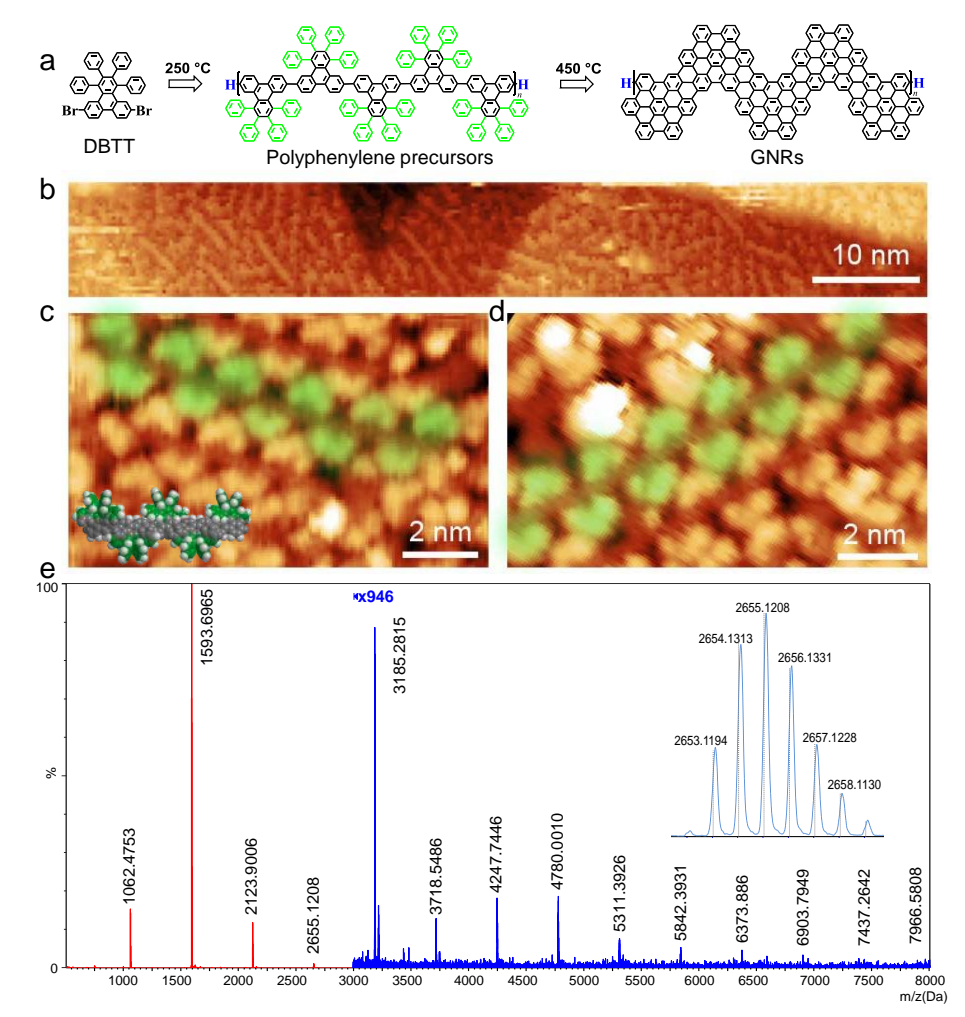

Figure 1. CVD growth of chevron-type GNRs and characterization of the intermediate polyphenylene precursors. a, CVD reaction scheme to chevron-type GNRs. b, $5 \mathrm{~K}$ UHV STM topography of the GNR polyphenylene precursors on Au/mica. c, d Zoom-in highlights undecameric and dodecameric polymer sections, where the tetraphenyl substitutions are marked in green (also the structure model in the inset of $\mathbf{c}$ and $\mathbf{a}$ ). Contamination adsorbed during ambient-pressure CVD (white protrusions) make high-resolution, long-range surveys challenging. $V_{\mathrm{t}}=1 \mathrm{~V}, I_{\mathrm{t}}=100$ pA. e, Mass spectrum of chevron-type polyphenylene precursors before cyclodehydrogenation, with a regular sequence of peaks from $\operatorname{dimer}(\mathrm{m} / \mathrm{z}=\sim 1,062 \mathrm{Da})$ to pentadecamer $(\mathrm{m} / \mathrm{z}=\sim 7,966 \mathrm{Da})$. Inset shows the isotopic distributions of the pentamer precursor, which agree well with the simulated results given in dotted lines. 

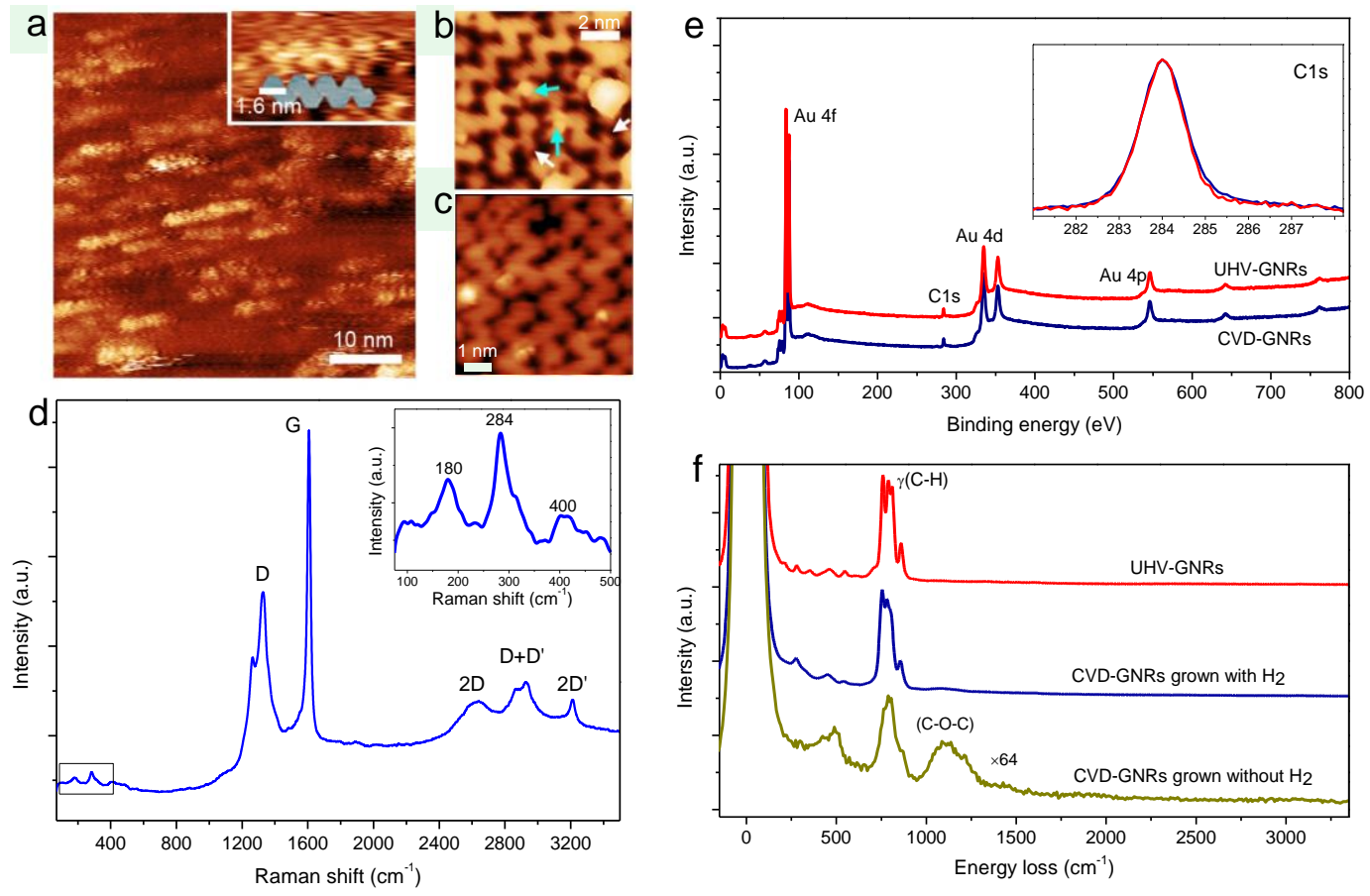

Figure 2. Microscopic and spectroscopic characterizations of chevron-type GNRs.

a, Ambient STM topography of the CVD-grown GNRs on Au/mica. A GNR model is also shown in the inset to scale. $V_{\mathrm{t}}=450 \mathrm{mV}, I_{\mathrm{t}}=15 \mathrm{pA}$. b-c, $5 \mathrm{~K}$ UHV STM data depicts the chevron-type structure. Arrows highlight common GNR imperfections. $V_{\mathrm{t}}$ $=1 \mathrm{~V}, I_{\mathrm{t}}=50 \mathrm{pA}$. d, Raman spectra of GNRs transferred on fused silica, measured at $532 \mathrm{~nm}$, the inset is the magnified low-frequency mode (black oblong, bottom left). e, f, XPS and HREELS spectra of the UHV-grown and CVD-grown GNRs. The HREELS spectra of CVD-GNRs grown without $\mathrm{H}_{2}$ is also showed in $\mathbf{f}$ for comparison. In the inset of $\mathbf{e}$, the $\mathrm{C} 1$ s core level spectra are presented. 

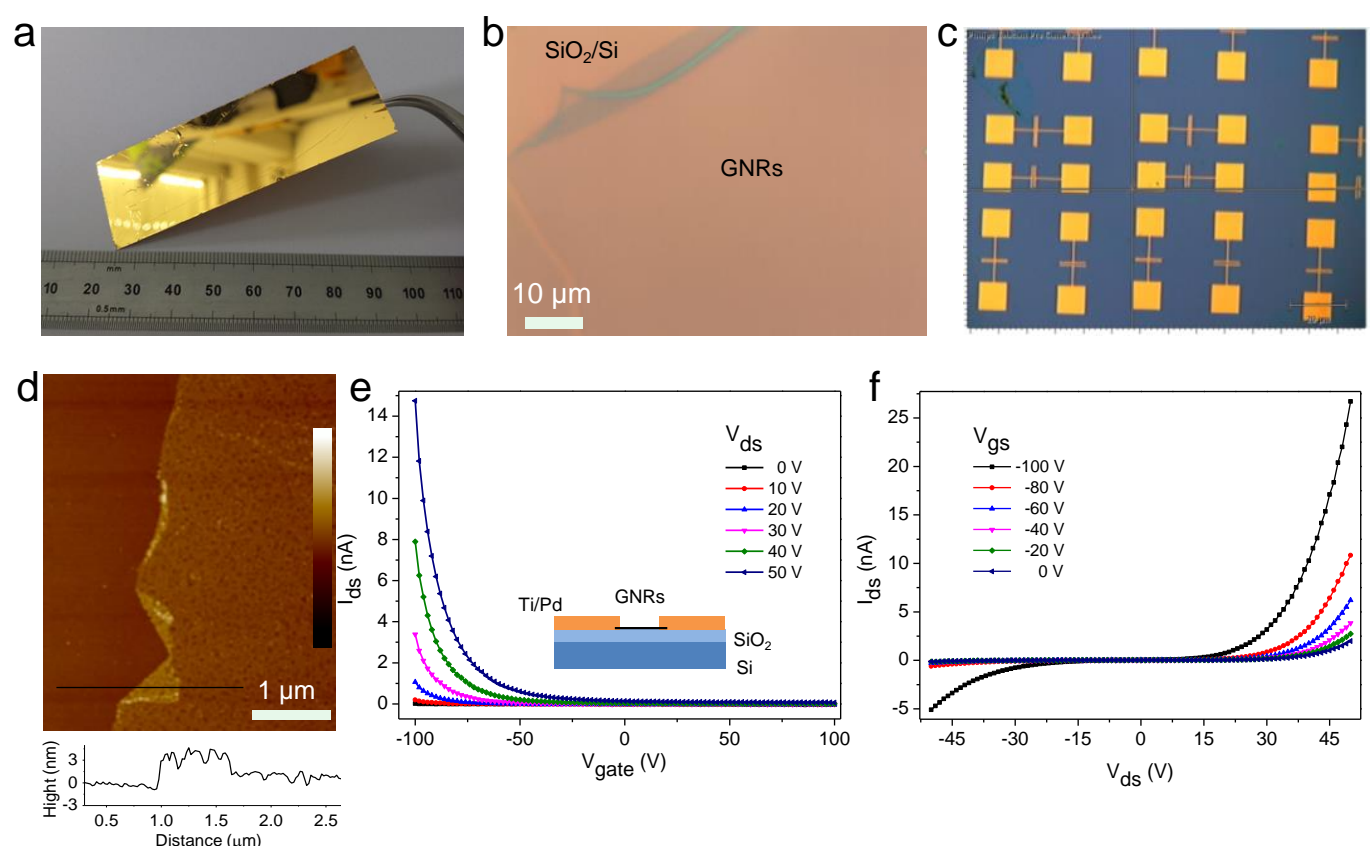

Figure 3. Transfer of CVD-grown chevron-type GNRs and micro-channel FET device fabrication. a, Photograph of a $25 \times 75 \mathrm{~mm}^{2}$ GNRs/Au/mica plate. $b$, Optical microscope image of the GNR film transferred on $\mathrm{SiO}_{2} / \mathrm{Si}$ substrate, showing the uniformity inside the film. The folding area can be found at the edge of the film. c, Optical microscope image of the devices built on GNR film. d, AFM topographical image of GNR film transferred on silicon wafer. The z-scale ranges from -16.5 to $16.5 \mathrm{~nm}$. The cross sectional high analysis is indicated along the black line. e, Transfer curves of a typical GNR thin film transistor measured at different $\mathrm{V}_{\mathrm{ds}}$. The results show that GNR transistors exhibit unipolar p-type behavior with on/off current ratio of $>1,000$. The highest on/off ratio we measured is $6,000 . \mathrm{f}, \mathrm{I}_{\mathrm{ds}}-\mathrm{V}_{\mathrm{ds}}$ curves of the GNR transistors. The curves display highly non-linear relationship, indicating a Schottky contact between electrodes and GNR channel. 

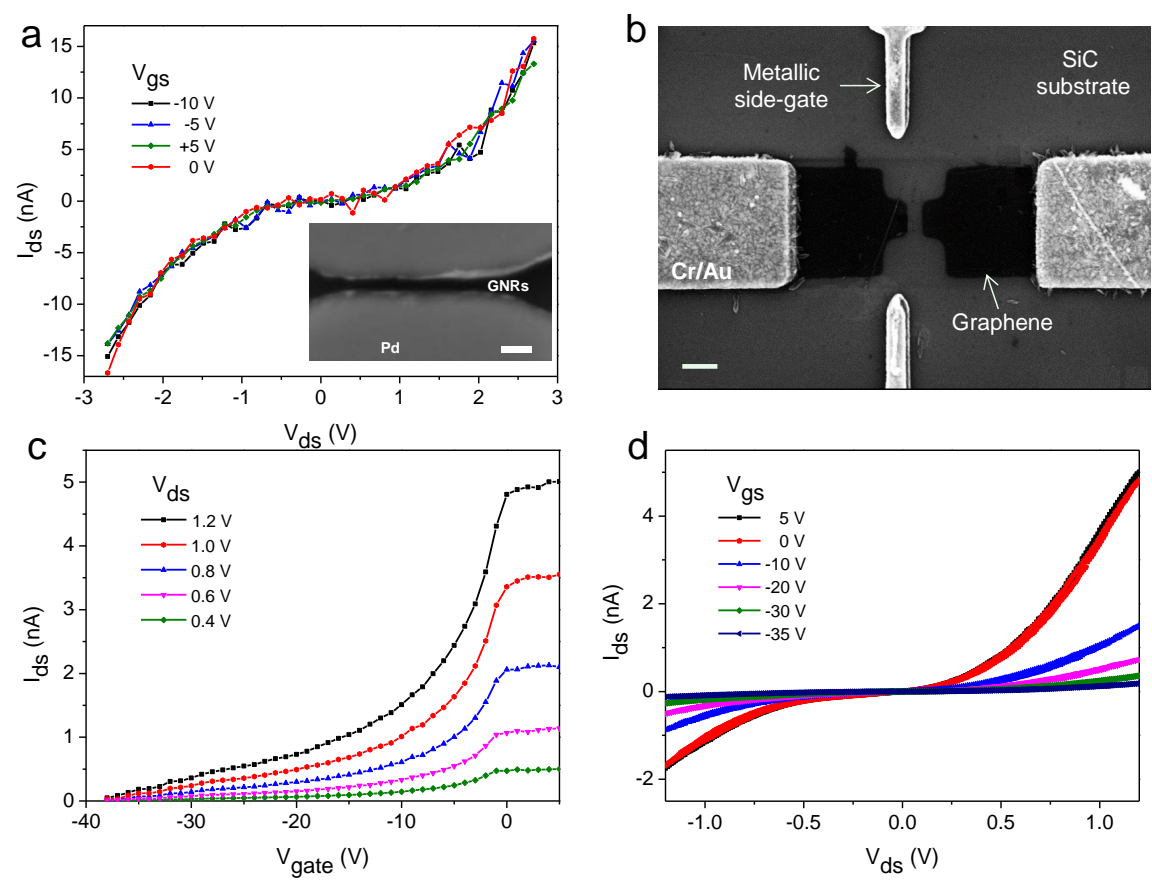

Figure 4. Electrical characteristics of nano-channel FET devices fabricated with CVD-grown chevron-type GNRs. a, $\mathrm{I}_{\mathrm{ds}}-\mathrm{V}_{\mathrm{ds}}$ curves of the short-channel devices at various gate voltages. The inset shows the scanning electron micrograph (SEM) of a typical short-channel device with an electrode spacing of $\sim 46 \mathrm{~nm}$. Scale bar, $100 \mathrm{~nm}$. b-d, Short-channel side-gate devices with graphene contacts. b, SEM image of the device on SiC substrate. Scale bar, $300 \mathrm{~nm}$. c, Transfer curves of a typical GNR device with graphene electrodes, exhibiting an n-type conduction with high current on/off ratios. $\mathbf{d}, \mathrm{I}_{\mathrm{ds}}-\mathrm{V}_{\mathrm{ds}}$ curves at different gate voltages. The non-linear behaviour of the $\mathrm{I}_{\mathrm{ds}}-\mathrm{V}_{\mathrm{ds}}$ curves indicates Schottky contacts with slightly asymmetric barriers. 
a
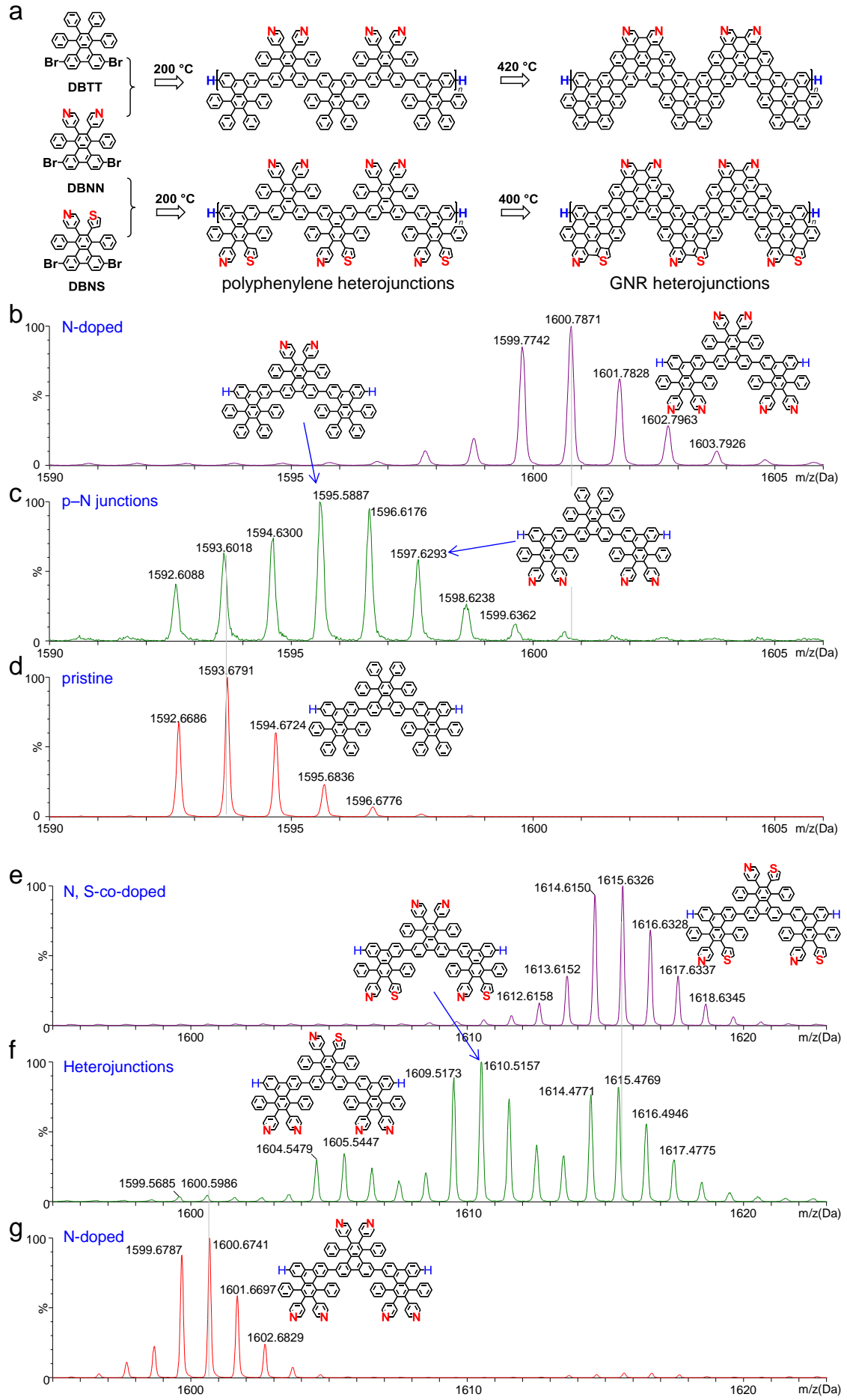

Figure 5. Heteroatom-doping and heterojunctions of polyphenylene precursors and GNRs. a, CVD reaction scheme to GNR heterojunctions by co-sublimation of DBTT/DBNN and DBNS/DBNN. b-d, Isotopic analysis of the N-doped (b) and pristine (d) polyphenylene trimers and their co-deposited $\mathrm{p}-\mathrm{N}$ polyphenylene heterojunctions (c) by high-resolution MALDI-TOF MS. e-g, Isotopic analysis of the $\mathrm{N}, \mathrm{S}$-co-doped (e) and N-doped polyphenylene trimers (g) and their co-deposited polyphenylene heterojunctions (f) by high-resolution MALDI-TOF MS. 
TOC
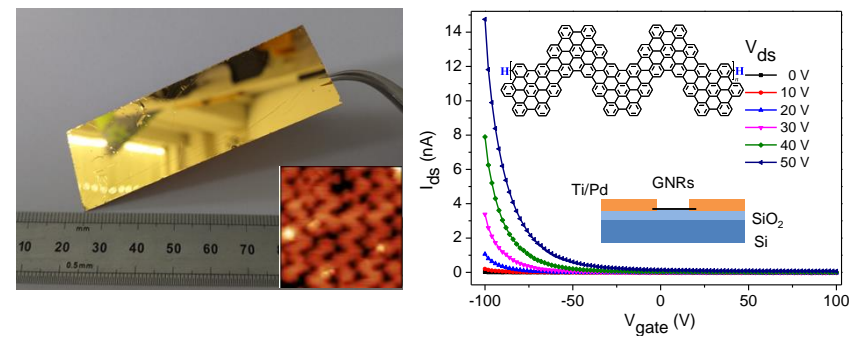

For Table of Contents Only 